\title{
Hyperreninemic Hypoaldosteronism
}

National Cancer Institute

\section{Source}

National Cancer Institute. Hyperreninemic Hypoaldosteronism. NCI Thesaurus. Code C126817.

Hypoaldosteronism characterized by impaired secretion of aldosterone, despite increased renin activity. This condition may be caused by chronic or critical illness, aldosterone synthase deficiency or other genetic conditions. 